\title{
COMMERCIAL AIRCRAFT ARINC429 DATA COLLECTING FROM AVIONICS SYSTEM INTEGRATION RIG
}

\author{
Quan Yiqing \\ Shanghai Aircraft Design and Research Institute \\ E-mail: quanyiqing@comac.cc
}

Keywords: RIG; ARINC429; Data bus; Label

\begin{abstract}
Currently, ARINC429 data buses are widely applied in commercial aircrafts for connecting avionic systems architecture and devices. The author does the research of collecting the avionics ARINC429 data and presents the method and suggestion.
\end{abstract}

\section{Introduction}

Avionics System Integration Rig is a ground-based engineering tool used to incorporate, optimize and validate vital aircraft systems, including flight controls, navigation and other avionics systems. It is the perfect tool to confirm the characteristics of all avionics system components, or to discover an incompatibility that may require modifications during early development stages. Additionally, the effects and subsequent treatment of failures introduced in the systems can be studied in full detail and recorded for analysis by using the Rig as a test bed. Specifically, Avionics System Integration Rig tends to simulate the whole avionics systems and focus on the entire aircraft cockpit. Most Line Replaceable Units (LRU) of avionics systems are installed in the rig cabinets.

Up to now, ARINC429 is still the most common data bus on the commercial aircrafts and their RIGs. Since it occurs on the Boeing aircrafts and Airbus aircrafts in the 1980s, hardly an avionics system on the aircraft has been developed that does not set up this data bus.

During the stage of avionic systems design and research, engineers have to monitor the status of data buses all the time for system testing, troubleshooting, software and hardware upgrades. So, collecting the ARINC429 data from RIG is a definitely important work.

\section{ARINC429 Data Bus}

ARINC429, "Mark33 Digital information Transfer System(DITS)" specification defines the air transport industry's standards for the transfer of digital data between avionics systems elements [1], including the physical and electrical interfaces of a two-wire data bus and a data protocol to support an aircraft's avionics local area network. This kind of predominant avionics data bus is used on most commercial and transport aircraft.

\section{Avionics System Integration RIG}

In this chapter, advanced digital avionics integration rig is introduced. With the rapid development of the electronic and computer technology, the major changes have been occurring in the design of avionics systems. Digital avionics, advanced multi-functional displays and data buses contribute to increase the automation, reliability and safety, which simplifies the aircraft crew operation.

Under this situation, the avionics systems are becoming complicated and hard to be certificated. Therefore, a ground based testing of the aircraft avionics is mandatory requirement for the certification process of any aircraft. The test schedules and the scope of the tests needs to include the condition these systems will operate real operation scenario.[2] 
The system integration rig is set up to integrate with various avionics subsystems and simulate the entire system architecture designed and also test all of these electrical and physical interfaces. Actually, according to the civil aviation regulation, all of the flight safety critical LRUs have to be tested on the system test platform, especially in system rig, before they are installed on the real aircraft. The avionics rig helps to perform the pre-installation checks and integration checks.

The rig usually includes powerful central computers which have the capability to process the inputs from the aircraft systems and output the simulation data to LRUs. The hardware of the simulation system will be installed on the system integration cabinet while the software hosting in the hardware (hard drives). Besides, the rig is always supplemented with an Iron Bird which is the mechanical devices used to simulate the aircraft control surfaces and other actuators or sensors.

\section{Method of Data Collecting}

This chapter provides a method of collecting the ARINC429 data from the RIG. There are many subsystems in the Rig including flight control systems, flight management systems, communication systems, navigation systems, landing gear system etc. Each subsystem comprises several labels of different functions. So, there are hundreds of labels and hundreds of thousands of data from the Rig. Therefore, before collecting the ARINC 429 data from the Rig, engineers should choose the data which they want. The author uses three key parameters of aircraft flight attitude - Pitch, Roll and Yaw as required data.

As shown from the Fig. 1, the author collects the data from the ARINC 429 patch panel, transmitting to the PC convertor by the special cable and then processed by the receiver. Finally, the readable data including aircraft attitude can be outputting to the microcontroller.

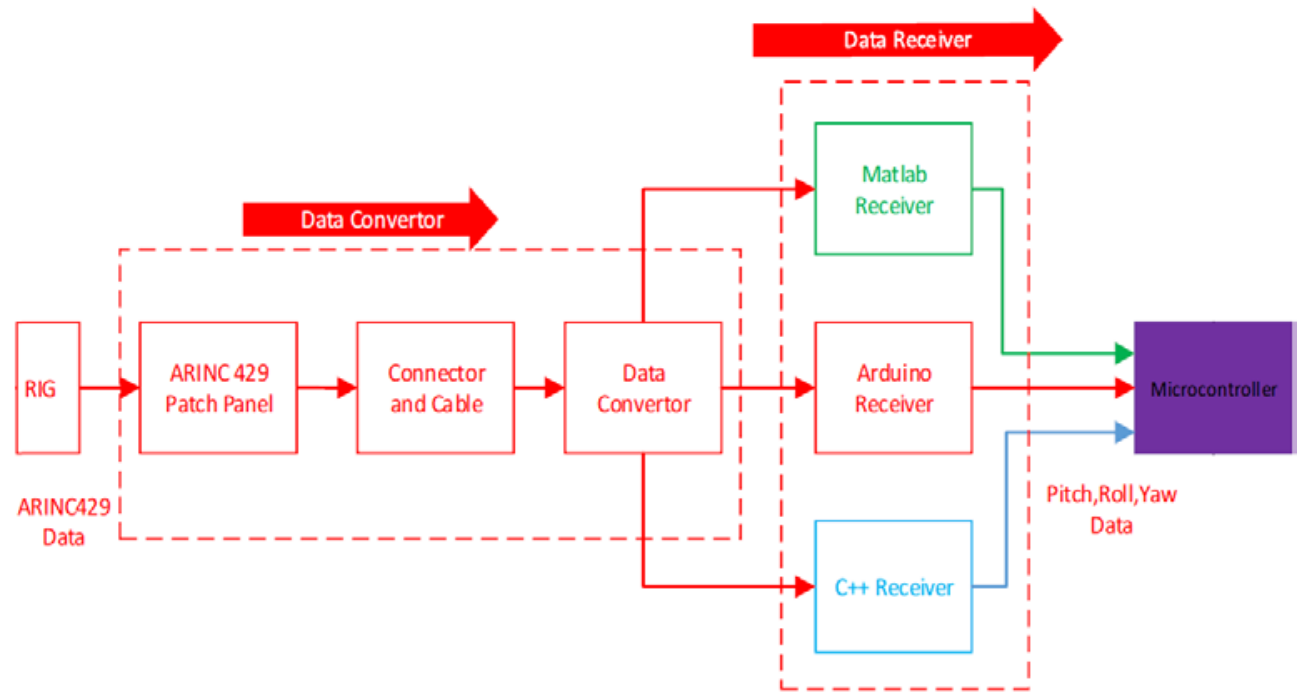

Fig. 1 Method of Data Collecting

\section{System Implementation}

According to the method of data collecting above, the author describes the system implementation in detail.

ARINC429 Data Convertor. In this section, the author will provide the method how to collect the ARINC 429 data from the Rig, and then convert it to the type of float which can be transmitted into the Ethernet. Fig. 2 shows the general view of ARINC data convertor. 


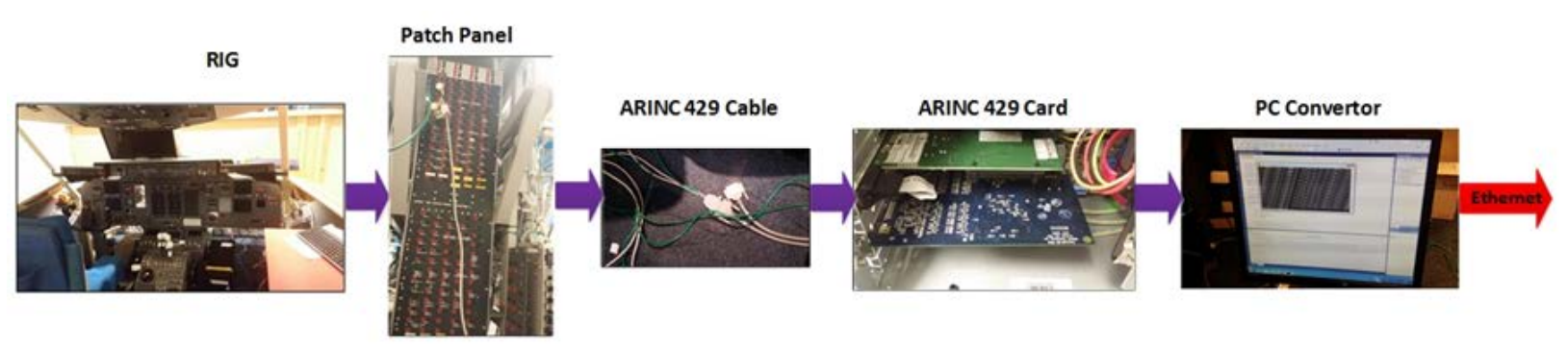

Fig. 2 ARINC Data Convertor

In the rig, a patch panel is also designed to interface with all the ARINC 429 parameter from these avionics systems and provide most of the avionics data from the data bus.. The patch panel is on the rear side of avionics rig and has multiple tapping points [2]. By this patch panel, the ARINC 429 data can be collected and then processed in the microcontroller for outputting. The aircraft attitude data comes from the ARINC data on the rig.

Generally, an ARINC 429 card is a peripheral component interconnect (PCI) compliant interface card capable of communicating over ARINC 429 avionics databuses. PCI is the common buses available in desktop computer. The connector and cable specially used for ARINC 429 data are made for collecting the data from the rig and then the data will be output into the computer which is installed one ARINC Card to process and convert the data.

All the ARINC 429 data of the main avionics subsystems will be processed and converted into the readable and recognizable parameters as Fig. 3.

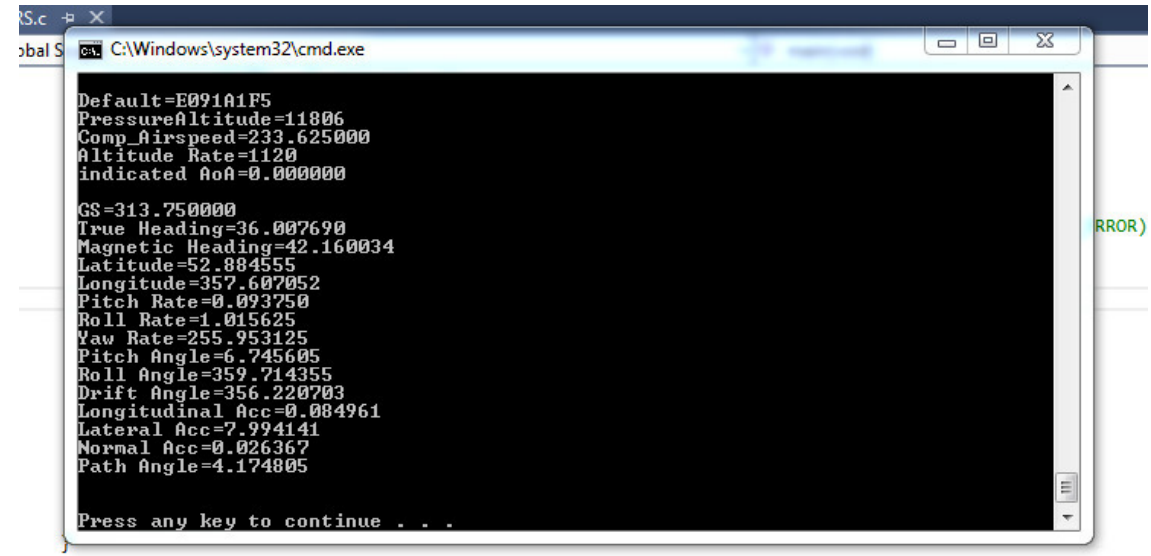

Fig. 3 Readable Parameters after Data Converting

Finally, the data will be transmitted into the Ethernet by the "float" data in that the users can choose anything they need for their own projects.

ARINC429 Data Receiver. After the data conversion, the author need to receive these data from the Ethernet. There are three methods to collect the data- $\mathrm{C}++$ (Microsoft visual studio), Matlab and Arduino Ethernet Shield.

For C++ (Microsoft Visual Studio), firstly, the author uses C++ on the Microsoft Visual Studio to receive the data from the Ethernet. The data is transmitted to the Ethernet by the float. Then, the author will receive the string data with the comma in each parameter. So, the next step is to delimit this string by the codes. Finally, the author choose and store the roll, pitch and yaw in the array by using "buffer []" and outputting to the microcontroller.

For Matlab and Simulink, Matlab and Simulink provide another way to achieve getting the readable parameters. Fig. 4 shows the method of collecting the data. 


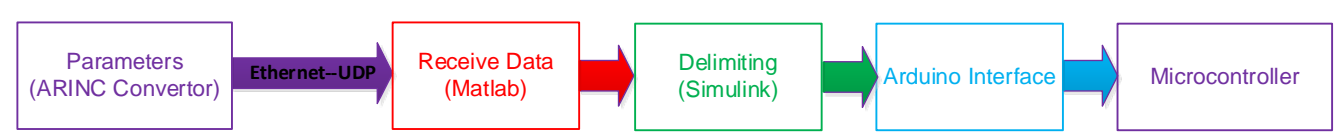

Fig. 4 Method of Collecting Data by Matlab

First of all, The PC on the RIG is computer A and the local PC is computer $\mathrm{B}$. The author gives the configuration in the Matlab. By the UDP function, codes on the Matlab allow the user to communicate and receive the data from the Ethernet. And then delimiting the string captured before the data is sent to Arduino interface. After that, configuring the Simulink and Collecting the Data from the Rig as Fig. 5. Finally, the Microcontroller can get the roll, pitch and yaw data from the rig. However, all of these above is just the test. When the user wants to collect the ARINC 429 data continuously, check the IP address of PC what connecting to the rig and then give the loop to the sections of delimiting the string and sending data to Simulink. After that, any data the user wants can be collected and then sent them to the Arduino interface in the Simulink communicating with the real Arduino microcontroller.

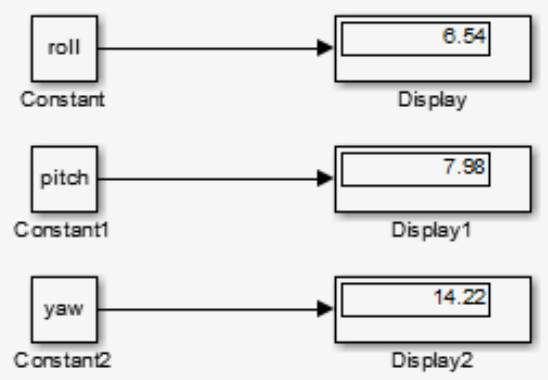

Fig. 5 Simulink Operating

For Arduino Ethernet Shield, Arduino Ethernet Shield is the simplest way to get the string data from the Ethernet directly. It can be stacked on the microcontroller such as Mega 2560 and connected to the Ethernet port by the cable. The codes for receiving the data is compiled. Then, the parameters are received. The next step is to delimit the string like the $\mathrm{C}++$ described above before outputting the data to the microcontroller.

In conclusion, by these three methods, the parameters can be received from the Ethernet and then outputting the roll, pitch and yaw data which the author wants to the microcontroller.

\section{Summary}

This article presents the method of collecting ARINC429 data from avionics system integration RIG. The author takes three parameters-Pitch, Roll and Yaw of aircraft flight attitude as an example, and then provides design procedure and system implementation in detail, finally, recommending three different methods of designing the data receivers. Through this research, it is beneficial for engineers to do the system research, test and troubleshooting during the avionic system design.

\section{References}

[1] ARINC SPECIFICATION 429PART1-16, ARINC, Published, September 27, 2001:1-2. [2]PRASHANTSINGH BHADORIA, Advanced Airframe \& Avionics Systems Integration Rig Commissioning \& Integrated Vehicle Health Management System, Cranfield University,2010:12. 